\title{
COVID-19 in Patients With Neuromyelitis Optica Spectrum Disorders and Myelin Oligodendrocyte Glycoprotein Antibody Disease in North America
}

\author{
From the COViMS Registry
}

Scott D. Newsome, DO, Anne H. Cross, MD, Robert J. Fox, MD, June Halper, MSN, APN-C, Pamela Kanellis, PhD, Bruce Bebo, PhD, David Li, MD, Gary R. Cutter, PhD, Kottil W. Rammohan, MD, and Amber Salter, PhD

Neurol Neuroimmunol Neuroinflamm 2021;8:e1057. doi:10.1212/NXI.0000000000001057

\section{Abstract}

\section{Background and Objective}

To describe the impact of coronavirus disease 2019 (COVID-19) on people with neuromyelitis optica spectrum disorders (NMOSD) and myelin oligodendrocyte glycoprotein antibody disease (MOGAD).

\section{Methods}

The COVID-19 Infections in Multiple Sclerosis (MS) and Related Diseases (COViMS) Registry collected data on North American patients with MS and related diseases with laboratory-positive or highly suspected SARS-CoV-2 infection. Deidentified data were entered into a web-based registry by health care providers. Data were analyzed using $t$-tests, Pearson $\chi^{2}$ tests, or Fisher exact tests for categorical variables. Univariate logistic regression models examined effects of risk factors and COVID-19 clinical severity.

\section{Results}

As of June 7, 2021, 77 patients with NMOSD and 20 patients with MOGAD were reported in the COViMS Registry. Most patients with NMOSD were laboratory positive for SARS-CoV-2 and taking rituximab at the time of COVID-19 diagnosis. Most patients with NMOSD were not hospitalized (64.9\% [95\% CI: 53.2\%-75.5\%]), whereas 15.6\% (95\% CI: 8.3\%-25.6\%) were hospitalized only, 9.1\% (95\% CI: 3.7\%-17.8\%) were admitted to the ICU and/or ventilated, and $10.4 \%$ (95\% CI: 4.6\%-19.5\%) died. In patients with NMOSD, having a comorbidity was the sole factor identified for poorer COVID-19 outcome (OR $=6.0,95 \%$ CI: 1.79-19.98). Most patients with MOGAD were laboratory positive for SARS-CoV-2, and almost half were taking rituximab. Among patients with MOGAD, 75.0\% were not hospitalized, and no deaths were recorded; no factors were different between those not hospitalized and those hospitalized, admitted to the ICU, or ventilated.

\section{Discussion}

Among the reported patients with NMOSD, a high mortality rate was observed, and the presence of comorbid conditions was associated with worse COVID-19 outcome. There were no deaths reported in the patients with MOGAD, although these observations are limited due to small sample size.
Correspondence Dr. Newsome snewsom2@jhmi.edu

\section{MORE ONLINE}

\section{COVID-19 Resources}

For the latest articles, invited commentaries, and blogs from physicians around the world

NPub.org/COVID 19

From the Johns Hopkins University School of Medicine (S.D.N.), Baltimore, MD; Washington University in St. Louis School of Medicine (A.H.C., A.S.), MO; Mellen Center for MS (R.J.F.), Cleveland Clinic, OH; Consortium of MS Centers (J.H.), Hackensack, NJ; MS Society of Canada (P.K.), Toronto, Ontario, Canada; National Multiple Sclerosis Society (B.B.) New York, NY; University of British Columbia (D.L.), Vancouver, British Columbia, Canada; The University of Alabama at Birmingham (G.R.C.); and University of Miami School of Medicine (K.W.R.), FL. 


\section{Glossary}

COVID-19 = coronavirus disease 2019; COViMS = COVID-19 Infections in MS and Related Diseases; DMT = diseasemodifying therapy; ICU = intensive care unit; MOGAD = myelin oligodendrocyte glycoprotein antibody disease; MS = multiple sclerosis; NMOSD = neuromyelitis optica spectrum disorders; SARS-CoV-2 = severe acute respiratory syndrome coronavirus 2 .

The coronavirus disease 2019 (COVID-19) pandemic caused by severe acute respiratory syndrome coronavirus 2 (SARSCoV-2) raised concerns about the risks of COVID-19 in those with neuroimmunologic disorders that affect the CNS. Risk factors associated with worse COVID-19 outcomes in multiple sclerosis (MS) include increased hospitalizations with B cell-depleting therapies, recent treatment with glucocorticoid use, and Black or African American race. ${ }^{1,2}$ However, little data have been reported on how COVID-19 affects people with neuromyelitis optica spectrum disorders (NMOSD) and myelin oligodendrocyte glycoprotein antibody disease (MOGAD).

NMOSD and MOGAD are distinct disorders that have a spectrum of presentations and unique autoantibodies that differentiate them from MS and other diseases. Disability levels vary in NMOSD and MOGAD, with the former often associated with a greater disability. However, outcomes of COVID19 in people with NMOSD or MOGAD are largely unknown, especially in those with preexisting medical comorbidities and/ or taking specific disease-modifying therapies (DMTs).

The rarity of coexisting SARS-CoV-2 and these autoantibodyassociated disorders make them well suited to a registry-based study. The COVID-19 Infections in MS and Related Diseases (COViMS) Registry was designed to collect information on COVID-19 in people with MS, NMOSD, and MOGAD from North American health care professionals. Here, we aimed to describe the clinical characteristics, risk factors for severe course of COVID-19, and overall COVID-19 outcomes in patients with NMOSD and MOGAD.

\section{Methods}

\section{Study Design}

The COViMS Registry collected data on patients in North America who had a laboratory positive or highly suspected infection with SARS-CoV-2 with MS and other related diseases, including NMOSD and MOGAD. ${ }^{1}$ Highly suspected patients for SARS-CoV-2 infection were identified by a health care professional based on typical COVID-19 symptoms and signs (e.g., anosmia, ageusia, shortness of breath, and pneumonia) with no better explanation for their symptoms/signs. Health care professionals were asked to report patients after a minimum of 7 days from initial infectious symptom onset and when sufficient time had passed to observe the COVID-19 disease course through resolution of acute illness or death. ${ }^{1}$ The COViMS Registry is jointly supported by the Consortium of MS Centers, the National MS Society USA, and the MS Society of Canada. ${ }^{1}$

Data collection was de-identified, cross-sectional, and entered into a secure, online database on the COViMS Registry website (COViMS.org) using the REDCap system (project-redcap. org). ${ }^{1}$ The Registry was determined to be not human subject research by the WUSTL Institutional Review Board. Data use agreements govern the use of some data that were contributed, and thus, access to individual level data is not permitted by outside researchers. ${ }^{1}$ Data collection began April 1, 2020, and is ongoing. ${ }^{1}$ The study herein used data collected from April 1, 2020, through June 7, 2021.

\section{COVID-19 Clinical Outcome}

Health care professionals indicated whether the patient was hospitalized, admitted to the intensive care unit (ICU), required ventilator support, and/or died during their COVID19 clinical course, with response options of "yes," "no," or "unknown" to each. ${ }^{1}$ Unknown responses were considered as not having the outcome. ${ }^{1}$ These separate outcomes were used to characterize COVID-19 severity, with the following levels: not hospitalized, hospitalization alone, ICU admission and/or ventilation and death where if more than 1 event was indicated, the patient was assigned to the highest level of event that occurred. ${ }^{1}$

\section{Demographic and Clinical Characteristics}

Demographic factors collected were sex (male, female, and nonbinary), age, race (White; Black or African American [Black]; Asian; American Indian, Alaska Native, or Indigenous Canadian; Native Hawaiian or Other Pacific Islander; other; or unknown), and ethnicity (Hispanic/Latino, not Hispanic/ Latino, other, or unknown). ${ }^{1}$

Clinical characteristics collected include disease type (if NMOSD, NMO-IgG or aquaporin-4 antibody status noted), disease duration, and ambulation milestones (fully ambulatory, walks with assistance, and nonambulatory). ${ }^{1}$ Comorbid conditions were indicated by selecting all applicable including cancer, cardiovascular disease, cerebrovascular disease, chronic kidney disease, chronic liver disease, chronic lung disease, chronic neurologic and/or neuromuscular disease, diabetes, hypertension, immunodeficiency disease, and morbid obesity. ${ }^{1}$ Comorbid conditions were also summed to categorize the count as 0 (reference), 1,2 , and $\geq 3$ comorbidities. ${ }^{1}$ Cigarette use history was captured as never, past, current, or unknown. ${ }^{1}$ Glucocorticoid treatment during the prior 2 months was ascertained. ${ }^{1}$ Current DMT at the time of SARS-CoV-2 
infection was reported by the provider as one of the following: alemtuzumab, azathioprine, cladribine, daclizumab, dimethyl fumarate, diroximel fumarate, eculizumab, fingolimod, glatiramer acetate, hematopoietic stem cell transplant, interferonbeta, intravenous immunoglobulin, methotrexate, mitoxantrone, mycophenolate, natalizumab, ocrelizumab, ofatumumab, ozanimod, rituximab, satralizumab, tocilizumab, siponimod, teriflunomide, other, none, and unknown. ${ }^{1}$

\section{Statistical Analysis}

The analyses were limited to patients with NMOSD and MOGAD who were laboratory positive or highly suspected for SARS-CoV-2 infection and reported by a health care professional in North America. Cohort characteristics were summarized using mean (SD) for continuous variables, median (interquartile range) for ordinal variables, and frequencies (\%) for categorical variables. ${ }^{1}$ Comparisons between groups were made using $\chi^{2}$ tests, Fisher exact tests, and Student $t$-tests, as appropriate, and reported descriptive statistics and $p$ values. ${ }^{1}$ Similar statistical comparisons were performed between patients with NMOSD and MS who died. Univariate logistic regression models were constructed to examine the effects of risk factors and COVID-19 clinical severity outcome dichotomized as those who were not hospitalized vs those hospitalized alone, admitted to the ICU and/or ventilated or death. ${ }^{1}$ The strength of associations was reported using OR and $95 \%$ CI. Statistical analyses were conducted using SAS V9.4 (SAS Institute Inc., Cary, NC). ${ }^{1}$

\section{Results}

\section{Demographic and Clinical Characteristics of Patients With NMOSD}

As of June 7, 2021, there were 77 patients with NMOSD reported in the COViMS Registry. Most patients were female $(81.6 \%)$ and in the United States at the time of COVID-19 (97.2\%), with a mean age (SD) of 48.1 (14.1) years (Table 1). Race and ethnicity of patients were diverse with $38.2 \%$ Black, $25.0 \%$ non-Hispanic White, $17.1 \%$ Hispanic or Latino, and $19.7 \%$ other racial groups. The average NMOSD disease duration was 9.1 (7) years, and $67.2 \%$ were aquaporin-4 IgG seropositive. Although the majority of patients with NMOSD were fully ambulatory (75.7\%), some required assistance with ambulation (14.9\%), and 9.4\% were nonambulatory. At the time of COVID-19 diagnosis, most patients with NMOSD were on rituximab (62.2\%), and 9.5\% were not on a DMT. Sixty percent had a comorbidity, with hypertension (20.8\%), diabetes (15.6\%), and morbid obesity (14.3\%) being the most frequent comorbidities reported. Only 4 (5.6\%) patients with NMOSD were current cigarette smokers.

Almost all (89.6\%) patients with NMOSD were laboratory positive for SARS-CoV-2. Fever (57.1\%) and dry cough (40.3\%) were commonly reported symptoms of COVID-19, whereas $2.6 \%$ of patients with NMOSD reported being asymptomatic. Seven patients reported having neurologic symptoms from COVID-19, with motor dysfunction occurring in 3 of the 7 and cognitive and sensory dysfunction each in 2 of the 7 patients. COVID-19 symptoms lasted 0-6 days in 13.3\% of patients with NMOSD, 7-13 days for 22.2\%, 14-20 days for $20.0 \%$, and 21 days or longer in $44.4 \%$ of patients. Of the 77 patients with NMOSD, $15.6 \%$ had pneumonia.

\section{COVID-19 Outcomes in Patients With NMOSD}

Most patients with NMOSD were not hospitalized (64.9\% [95\% CI: $53.2 \%-75.5 \%]$ ), whereas $15.6 \%$ (95\% CI: $8.3 \%-25.6 \%$ ) were hospitalized only, and 9.1\% (95\% CI: $3.7 \%-17.8 \%$ ) were admitted to the ICU and/or ventilated. Over 10\% (10.4\%; $95 \%$ CI: $4.6 \%-19.5 \%)$ died. No demographic characteristics were statistically significantly different between those not hospitalized and those hospitalized, admitted to the ICU, ventilated, or died of COVID-19. Having a medical comorbidity was the only statistically significant difference observed; those with a comorbidity were 6-fold more likely to have a poor clinical COVID-19 outcome compared with those with no comorbidity $(\mathrm{OR}=6.0$, 95\% CI: 1.79-19.98).

A description of the demographic and clinical characteristics of those 8 patients with NMOSD who died of COVID-19 is reported in Table 2. All deceased patients with NMOSD had at least 1 medical comorbidity. Five of the 8 (62.5\%) fatal NMOSD cases were aquaporin-4 IgG positive, and 4 (50\%) were Black or African American. Six (75.0\%) of the 8 patients with NMOSD who died of COVID-19 were on rituximab, but the proportion of deaths among those taking rituximab was not statistically different than the proportion among those not on rituximab (6/ $46=13.0 \%$ compared with $2 / 31=6.5 \%, p=0.46$ ). No significant differences were found among those taking rituximab vs those not on rituximab among patients with NMOSD who were hospitalized or worse $(6 / 18=33.3 \%$ taking rituximab compared with $2 / 9=29.0 \%$ not taking rituximab, $p=0.68$ ).

\section{Demographic and Clinical Characteristics of Patients With MOGAD}

Twenty patients with MOGAD with COVID-19 were reported in the COViMS Registry. Most patients were female (70.0\%) with a mean (SD) age of 40.6 (18.7) years. One half identified as non-Hispanic White. The average disease duration was 5.4 (5.0) years, and most patients with MOGAD were fully ambulatory (84.2\%). Almost half of the patients with MOGAD were on rituximab at the time of their COVID-19 diagnosis. Forty-seven percent had a comorbidity, with hypertension (25.0\%), diabetes (15.0\%), and morbid obesity (15.0\%) being the most common. Most patients were laboratory positive (95.0\%) for SARS-CoV-2. The most common symptoms from COVID-19 reported in these patients with MOGAD were fever $(60.0 \%)$, fatigue (55.0\%), dry cough (45.0\%), and shortness of breath (4.0.\%). The symptom duration was $0-14$ days in $25.0 \%$ of patients with MOGAD, 14-20 days in 35.0\%, 21 days or longer in $10.0 \%$, and unknown in $30.0 \%$. Pneumonia was reported in $4(20.0 \%)$ patients.

\section{COVID-19 Outcomes in Patients With MOGAD}

Of the patients with MOGAD, $75.0 \%$ (95\% CI: 50.9\%-91.3\%) were not hospitalized, $10.0 \%$ (95\% CI: $1.2 \%-31.7 \%$ ) were 
Table 1 COViMS NMOSD Overall and by Clinical Outcome

\begin{tabular}{|c|c|c|c|c|}
\hline Factor & Total $(\mathbf{N}=\mathbf{7 7})$ & $\begin{array}{l}\text { Not hospitalized } \\
(n=50)\end{array}$ & $\begin{array}{l}\text { Hospitalized, } \\
\text { ICU admission/ } \\
\text { ventilation/death } \\
(n=27)\end{array}$ & $p$ Value \\
\hline Female & $62(81.6)$ & $43(86.0)$ & $19(73.1)$ & $0.17^{\mathrm{b}}$ \\
\hline Age & $48.1(14.1)$ & $46.9(14.1)$ & $50.3(14.2)$ & $0.33^{\mathrm{e}}$ \\
\hline Race & & & & $0.13^{b}$ \\
\hline Non-Hispanic White & $19(25.0)$ & $11(22.4)$ & $8(29.6)$ & \\
\hline Black or African American & $29(38.2)$ & $18(36.7)$ & $11(40.7)$ & \\
\hline Hispanic or Latino & $13(17.1)$ & $12(24.5)$ & $1(3.7)$ & \\
\hline Other/unknown & $15(19.7)$ & $8(16.3)$ & $7(25.9)$ & \\
\hline Disease duration $^{c}$ & $9.1(7.0)$ & $8.6(6.5)$ & $10.2(7.9)$ & $0.37^{a}$ \\
\hline NMOSD type & & & & $0.47^{\mathrm{b}}$ \\
\hline NMO-IgG/aquaporin-4 antibody (+) & $43(55.8)$ & $28(56.0)$ & $15(55.6)$ & \\
\hline NMO-IgG/aquaporin-4 antibody (-) & $15(19.5)$ & $8(16.0)$ & $7(25.9)$ & \\
\hline Unknown & $19(24.7)$ & $14(28.0)$ & $5(18.5)$ & \\
\hline Ambulatory status & & & & $0.21^{d}$ \\
\hline Fully ambulatory & $56(75.7)$ & $40(81.6)$ & $16(64.0)$ & \\
\hline Walk with assistance & $11(14.9)$ & $6(12.2)$ & $5(20.0)$ & \\
\hline Nonambulatory & $7(9.5)$ & $3(6.1)$ & $4(16.0)$ & \\
\hline Glucocorticoid during the last 2 mor & & & & $0.67^{d}$ \\
\hline No & $56(72.7)$ & $36(72.0)$ & $20(74.1)$ & \\
\hline Yes & $6(7.8)$ & $5(10.0)$ & $1(3.7)$ & \\
\hline Unknown & $15(19.5)$ & $9(18.0)$ & $6(22.2)$ & \\
\hline DMT at the time of COVID-19 ${ }^{c}$ & & & & $0.63^{d}$ \\
\hline Azathioprine & $5(6.8)$ & $4(8.5)$ & $1(3.7)$ & \\
\hline Eculizumab & $5(6.8)$ & $4(8.5)$ & $1(3.7)$ & \\
\hline Mycophenolate & $5(6.8)$ & $4(8.5)$ & $1(3.7)$ & \\
\hline Ocrelizumab & $1(1.4)$ & $0(0.0)$ & $1(3.7)$ & \\
\hline Rituximab & $46(62.2)$ & $28(59.6)$ & $18(66.7)$ & \\
\hline Other & $5(6.8)$ & $2(4.3)$ & $3(11.1)$ & \\
\hline None & $7(9.5)$ & $5(10.6)$ & $2(7.4)$ & \\
\hline Length of time on current $\mathrm{DMT}^{\mathrm{c}}$ & & & & $0.49^{\mathrm{b}}$ \\
\hline$<6$ months & & & & $0.32^{\mathrm{b}}$ \\
\hline 6 or more months & $9(13.8)$ & $7(17.1)$ & $2(8.3)$ & \\
\hline Have comorbidities? & $56(86.2)$ & $34(82.9)$ & $22(91.7)$ & \\
\hline No & & & & $0.004^{b}$ \\
\hline Yes & $29(38.7)$ & $25(52.1)$ & $4(14.8)$ & \\
\hline Cancer & $5(6.5)$ & $2(4.0)$ & $3(11.1)$ & $0.34^{d}$ \\
\hline Cardiovascular disease & $6(7.8)$ & $4(8.0)$ & $2(7.4)$ & $0.99^{d}$ \\
\hline Cerebrovascular disease & $1(1.3)$ & $1(2.0)$ & $0(0.0)$ & $0.99^{d}$ \\
\hline
\end{tabular}


Table 1 COViMS NMOSD Overall and by Clinical Outcome (continued)

\begin{tabular}{|c|c|c|c|c|}
\hline Factor & Total $(\mathbf{N}=\mathbf{7 7})$ & $\begin{array}{l}\text { Not hospitalized } \\
(n=50)\end{array}$ & $\begin{array}{l}\text { Hospitalized, } \\
\text { ICU admission/ } \\
\text { ventilation/death } \\
(n=27)\end{array}$ & $p$ Value \\
\hline Chronic kidney disease & $2(2.6)$ & $0(0.0)$ & $2(7.4)$ & $0.12^{\mathrm{d}}$ \\
\hline Chronic liver disease & $0(0.0)$ & $0(0.0)$ & $0(0.0)$ & \\
\hline Chronic lung disease & $7(9.1)$ & $3(6.0)$ & $4(14.8)$ & $0.23^{d}$ \\
\hline Chronic neurologic disease & $4(5.2)$ & $1(2.0)$ & $3(11.1)$ & $0.12^{\mathrm{d}}$ \\
\hline Diabetes & $12(15.6)$ & $7(14.0)$ & $5(18.5)$ & $0.60^{\mathrm{b}}$ \\
\hline Hypertension & $16(20.8)$ & $9(18.0)$ & 7 (25.9) & $0.41^{\mathrm{b}}$ \\
\hline Immunodeficiency disease & $2(2.6)$ & $1(2.0)$ & $1(3.7)$ & $0.99^{d}$ \\
\hline Morbid obesity & $11(14.3)$ & $4(8.0)$ & $7(25.9)$ & $0.032^{\mathrm{b}}$ \\
\hline Other & $12(15.6)$ & $8(16.0)$ & $4(14.8)$ & $0.89^{\mathrm{b}}$ \\
\hline Comorbid conditions count & & & & $0.027^{b}$ \\
\hline 0 & $32(41.6)$ & $27(54.0)$ & $5(18.5)$ & \\
\hline 1 & $24(31.2)$ & $12(24.0)$ & $12(44.4)$ & \\
\hline 2 & $10(13.0)$ & $5(10.0)$ & $5(18.5)$ & \\
\hline$\geq 3$ & $11(14.3)$ & $6(12.0)$ & $5(18.5)$ & \\
\hline Type of COVID-19 diagnosis & & & & $0.028^{b}$ \\
\hline Laboratory positive & $69(89.6)$ & $42(84.0)$ & $27(100.0)$ & \\
\hline Suspected COVID-19, not confirmed & $8(10.4)$ & $8(16.0)$ & $0(0.0)$ & \\
\hline \multicolumn{5}{|c|}{$\begin{array}{l}\text { Abbreviations: COVID-19 = coronavirus disease 2019; COViMS = COVID-19 Infections in MS and Related Diseases; DMT = disease-modifying therapy; ICU = } \\
\text { intensive care unit; NMOSD = neuromyelitis optica spectrum disorders. } \\
\text { Values presented as mean } \pm \text { SD, median (P25, P75) or N (column \%). } \\
\text { a Pearson } \chi^{2} \text { test. } \\
\text { b ANOVA. } \\
\text { ' Data not available for all patients. Missing values: sex =1, age =1, disease duration = 5, DMT at the time of COVID-19=2, and length of time on current DMT = } \\
10 \text {. } \\
\text { ' Fisher exact test. } \\
\text { ' } \text { Kruskal-Wallis test. }\end{array}$} \\
\hline
\end{tabular}

hospitalized, and 15.0\% (95\% CI: 3.2\%-37.9\%) were admitted to the ICU and/or ventilated. No deaths were recorded as of June 7, 2021. A full listing of individual MOGAD patient clinical characteristics, COVID-19 clinical outcome, and COVID-19 treatments is provided in Table 3. No demographic characteristic or clinical COVD-19 outcome was statistically different between those not hospitalized and those hospitalized, admitted to the ICU, or ventilated (data not shown and available on request). Notably, these comparisons were limited due to the small number of patients with MOGAD who were reported.

\section{Differences in COVID-19 Deaths Among Patients With NMOSD and MS}

Seventy-four patients with MS and 8 patients with NMOSD died during the study time period. There were a few differences in demographic and clinical characteristics between these groups worth noting (Table 4). Specifically, the NMOSD cohort who died were younger $(50 \pm 19.2$ vs $62.1 \pm 12.3$, $p=0.016)$, had shorter disease duration ( $9.3 \pm 7.4$ vs $20.6 \pm 12.1, p=0.012)$, and a greater percentage of patients were on rituximab compared with the patients with MS who died (75\% vs $5.4 \%, p<0.001$ ).

\section{Discussion}

The COViMS Registry has provided a platform for collecting clinician-reported data on patients with MS and related disorders who developed COVID-19. These Registry data provide clinicians with useful information about how SARS-CoV-2 infection affects patients with NMOSD and MOGAD and indicate those who might be at greatest risk for more severe COVID-19 outcomes. NMOSD and MOGAD are uncommon, and this study represents the largest cohort of patients with NMOSD and MOGAD with COVID-19 reported to date. A French study of 15 total patients with NMOSD and MOGAD (5 seropositive NMOSD, 5 seronegative NMOSD, and 5 with MOGAD) with COVID-19 reported an association of older age and more disability with hospitalization, but no deaths. ${ }^{3}$ 
Table 2 Demographic and Clinical Characteristics of Deceased Patients With NMOSD

\begin{tabular}{|c|c|c|c|c|c|c|c|c|c|c|c|}
\hline Sex & Age & Race & $\begin{array}{l}\text { US state at the } \\
\text { time of COVID-19 } \\
\text { onset }\end{array}$ & $\begin{array}{l}\text { Disease } \\
\text { duration } \\
\text { (y) }\end{array}$ & $\begin{array}{l}\text { NMOSD } \\
\text { antibody } \\
\text { serostatus }\end{array}$ & $\begin{array}{l}\text { Ambulatory } \\
\text { status }\end{array}$ & $\begin{array}{l}\text { DMT at the } \\
\text { time of } \\
\text { COVID-19 }\end{array}$ & Comorbidities & Pneumonia & $\begin{array}{l}\text { Length of } \\
\text { hospital } \\
\text { stay }\end{array}$ & COVID-19 treatments \\
\hline Female & 73 & $\begin{array}{l}\text { Non- } \\
\text { Hispanic } \\
\text { White }\end{array}$ & Colorado & 2 & Positive & $\begin{array}{l}\text { Walk with } \\
\text { assistance }\end{array}$ & None & $\begin{array}{l}\text { Cancer, cardiovascular } \\
\text { disease, and chronic lung } \\
\text { disease }\end{array}$ & Yes & $4-6 d$ & $\begin{array}{l}\text { Hydroxychloroquine, azithromycin, and oxygen } \\
\text { therapy }\end{array}$ \\
\hline Female & 30 & $\begin{array}{l}\text { Black or } \\
\text { African } \\
\text { American }\end{array}$ & Florida & 2 & Negative & $\begin{array}{l}\text { Fully } \\
\text { ambulatory }\end{array}$ & Rituximab & $\begin{array}{l}\text { Diabetes and morbid } \\
\text { obesity }\end{array}$ & Yes-bilateral & $19-21 d$ & $\begin{array}{l}\text { Antiviral and other antibiotic, convalescent } \\
\text { plasma, tocilizumab, and oxygen therapy }\end{array}$ \\
\hline Female & 55 & $\begin{array}{l}\text { Other/ } \\
\text { unknown }\end{array}$ & Unknown & 9 & Positive & $\begin{array}{l}\text { Walk with } \\
\text { assistance }\end{array}$ & Rituximab & Hypertension & Unknown & $30+d$ & Unknown \\
\hline Female & 39 & $\begin{array}{l}\text { Black or } \\
\text { African } \\
\text { American }\end{array}$ & Washington & 10 & Positive & Nonambulatory & Other & $\begin{array}{l}\text { Chronic kidney disease } \\
\text { and morbid obesity }\end{array}$ & Unknown & $4-6 d$ & Unknown \\
\hline Female & 26 & $\begin{array}{l}\text { Black or } \\
\text { African } \\
\text { American }\end{array}$ & California & 5 & Positive & $\begin{array}{l}\text { Fully } \\
\text { ambulatory }\end{array}$ & Rituximab & $\begin{array}{l}\text { Diabetes and morbid } \\
\text { obesity }\end{array}$ & Yes & Unknown & Unknown \\
\hline Male & 80 & $\begin{array}{l}\text { Non- } \\
\text { Hispanic } \\
\text { White }\end{array}$ & Missouri & 25 & Negative & Nonambulatory & Rituximab & Hypertension & No & $7-9 d$ & Unknown \\
\hline Female & 46 & $\begin{array}{l}\text { Non- } \\
\text { Hispanic } \\
\text { White }\end{array}$ & Vermont & 8 & Negative & $\begin{array}{l}\text { Fully } \\
\text { ambulatory }\end{array}$ & Rituximab & $\begin{array}{l}\text { Diabetes, hypertension, } \\
\text { and morbid obesity }\end{array}$ & Yes-bilateral & $30+d$ & $\begin{array}{l}\text { Antiviral, systemic glucocorticoids, other anti- } \\
\text { inflammatory agents, oxygen therapy, IVIG, and } \\
\text { convalescent plasma }\end{array}$ \\
\hline Female & 52 & $\begin{array}{l}\text { Black or } \\
\text { African } \\
\text { American }\end{array}$ & Kentucky & 13 & Positive & $\begin{array}{l}\text { Fully } \\
\text { ambulatory }\end{array}$ & Rituximab & $\begin{array}{l}\text { Chronic lung disease and } \\
\text { hypertension }\end{array}$ & Yes-bilateral & $30+d$ & $\begin{array}{l}\text { Systemic glucocorticoids, azithromycin, other } \\
\text { antibiotics, oxygen therapy, and IVIG }\end{array}$ \\
\hline
\end{tabular}

Abbreviations: COVID-19 = coronavirus disease 2019; DMT = disease-modifying therapy; IVIG = intravenous immunoglobulin; NMOSD = neuromyelitis optica spectrum disorders. 


\begin{tabular}{|c|c|c|c|c|c|c|c|c|c|c|c|}
\hline Sex & Age & Race & $\begin{array}{l}\text { US state at the time of } \\
\text { COVID-19 onset }\end{array}$ & $\begin{array}{l}\text { Disease } \\
\text { duration }\end{array}$ & $\begin{array}{l}\text { Ambulatory } \\
\text { status }\end{array}$ & $\begin{array}{l}\text { DMT at the time } \\
\text { of COVID-19 }\end{array}$ & Comorbidities & $\begin{array}{l}\text { COVID-19 clinical } \\
\text { severity outcome }\end{array}$ & Pneumonia & $\begin{array}{l}\text { Length of } \\
\text { hospital stay }\end{array}$ & COVID-19 treatments \\
\hline Female & 67 & $\begin{array}{l}\text { Black or } \\
\text { African } \\
\text { American }\end{array}$ & Unknown & 15 & $\begin{array}{l}\text { Fully } \\
\text { ambulatory }\end{array}$ & Mycophenolate & $\begin{array}{l}\text { Cancer, chronic lung } \\
\text { disease, and diabetes }\end{array}$ & $\begin{array}{l}\text { Admitted to the ICU } \\
\text { and/or ventilated }\end{array}$ & No & $13-15 d$ & $\begin{array}{l}\text { Hydroxychloroquine and } \\
\text { systemic glucocorticoids }\end{array}$ \\
\hline Male & 54 & $\begin{array}{l}\text { Non-Hispanic } \\
\text { White }\end{array}$ & Kansas & 3 & $\begin{array}{l}\text { Fully } \\
\text { ambulatory }\end{array}$ & Rituximab & Hypertension & $\begin{array}{l}\text { Admitted to the ICU } \\
\text { and/or ventilated }\end{array}$ & Yes-bilateral & $7-9 d$ & $\begin{array}{l}\text { Chloroquine, azithromycin, and } \\
\text { oxygen therapy }\end{array}$ \\
\hline Female & 19 & $\begin{array}{l}\text { Non-Hispanic } \\
\text { White }\end{array}$ & California & 3 & $\begin{array}{l}\text { Fully } \\
\text { ambulatory }\end{array}$ & Mycophenolate & No & Hospitalization only & Yes-bilateral & $4-6 d$ & $\begin{array}{l}\text { Hydroxychloroquine, } \\
\text { azithromycin, and other } \\
\text { antibiotics }\end{array}$ \\
\hline Female & 26 & $\begin{array}{l}\text { Non-Hispanic } \\
\text { White }\end{array}$ & $\begin{array}{l}\text { Unknown-United } \\
\text { States }\end{array}$ & 2 & $\begin{array}{l}\text { Fully } \\
\text { ambulatory }\end{array}$ & Rituximab & Obesity & Not hospitalized & No & - & None \\
\hline Female & 58 & $\begin{array}{l}\text { Hispanic or } \\
\text { Latino }\end{array}$ & Missouri & 7 & $\begin{array}{l}\text { Fully } \\
\text { ambulatory }\end{array}$ & None & No & Not hospitalized & No & - & Systemic glucocorticoids \\
\hline Female & 32 & $\begin{array}{l}\text { Other/ } \\
\text { unknown }\end{array}$ & California & 3 & $\begin{array}{l}\text { Fully } \\
\text { ambulatory }\end{array}$ & Rituximab & Morbid obesity & Not hospitalized & No & - & Azithromycin \\
\hline Female & 43 & $\begin{array}{l}\text { Non-Hispanic } \\
\text { White }\end{array}$ & Kentucky & 2 & $\begin{array}{l}\text { Walk with } \\
\text { assistance }\end{array}$ & Azathioprine & $\begin{array}{l}\text { Diabetes, hypertension, } \\
\text { and morbid obesity }\end{array}$ & Not hospitalized & Yes-unilateral & - & Azithromycin \\
\hline Female & 20 & $\begin{array}{l}\text { Hispanic or } \\
\text { Latino }\end{array}$ & $\begin{array}{l}\text { Unknown-United } \\
\text { States }\end{array}$ & 8 & $\begin{array}{l}\text { Fully } \\
\text { ambulatory }\end{array}$ & Fingolimod & Migraines & Not hospitalized & No & - & None \\
\hline Female & 62 & $\begin{array}{l}\text { Other/ } \\
\text { unknown }\end{array}$ & California & 2 & $\begin{array}{l}\text { Fully } \\
\text { ambulatory }\end{array}$ & Rituximab & None & Not hospitalized & No & - & Tylenol \\
\hline Female & 26 & $\begin{array}{l}\text { Non-Hispanic } \\
\text { White }\end{array}$ & Rhode Island & 2 & $\begin{array}{l}\text { Fully } \\
\text { ambulatory }\end{array}$ & Rituximab & No & Not hospitalized & No & - & Unknown \\
\hline Female & 64 & $\begin{array}{l}\text { Other/ } \\
\text { unknown }\end{array}$ & $\begin{array}{l}\text { Unknown-United } \\
\text { States }\end{array}$ & 0 & $\begin{array}{l}\text { Walk with } \\
\text { assistance }\end{array}$ & Rituximab & $\begin{array}{l}\text { Diabetes, hypertension, } \\
\text { hyperlipidemia, and } \\
\text { obstructive sleep apnea }\end{array}$ & Not hospitalized & No & - & None \\
\hline Female & 25 & $\begin{array}{l}\text { Non-Hispanic } \\
\text { White }\end{array}$ & Nebraska & 15 & $\begin{array}{l}\text { Fully } \\
\text { ambulatory }\end{array}$ & IVIG & None & Not hospitalized & No & - & Bamlanivimab \\
\hline Male & 69 & $\begin{array}{l}\text { Other/ } \\
\text { unknown }\end{array}$ & Canada & 4 & $\begin{array}{l}\text { Fully } \\
\text { ambulatory }\end{array}$ & None & Cancer and hypertension & Not hospitalized & No & - & None \\
\hline Male & 58 & $\begin{array}{l}\text { Non-Hispanic } \\
\text { White }\end{array}$ & Ohio & 2 & $\begin{array}{l}\text { Fully } \\
\text { ambulatory }\end{array}$ & Rituximab & $\begin{array}{l}\text { Hypertension and } \\
\text { morbid obesity }\end{array}$ & $\begin{array}{l}\text { Admitted to the ICU } \\
\text { and/or ventilated }\end{array}$ & Yes-bilateral & $1-3 d$ & $\begin{array}{l}\text { Unknown monoclonal antibody } \\
\text { infusion }\end{array}$ \\
\hline Female & 27 & $\begin{array}{l}\text { Non-Hispanic } \\
\text { White }\end{array}$ & Canada & 4 & $\begin{array}{l}\text { Fully } \\
\text { ambulatory }\end{array}$ & Azathioprine & None & Not hospitalized & No & - & None \\
\hline Male & 27 & $\begin{array}{l}\text { Other/ } \\
\text { unknown }\end{array}$ & Canada & Unknown & Unknown & Unknown & None & Not hospitalized & No & - & Unknown \\
\hline
\end{tabular}


Approximately two-thirds of patients with NMOSD in the COViMS Registry did not require hospitalization; however, $10.4 \%$ of the entire cohort died (8/77). This mortality rate is notable for being 4 times the rate than what was seen with patients with MS in COViMS and almost 6 times greater than that reported for the general population in the United States. ${ }^{1,4}$ However, a $95 \%$ CI ranging from $4.6 \%$ to $19.5 \%$ leaves some uncertainty as to the level of excess risk. A large percentage of the NMOSD cohort were Black and on rituximab, both of which are known risk factors for severe COVID19 in other populations. ${ }^{1,2,5}$ The sole factor that identified statistically poorer COVID-19 outcome in NMOSD was the presence of a medical comorbidity, which resulted in 6-fold greater odds of worse COVID-19 outcomes. The most common medical comorbidities in this cohort were hypertension, diabetes, and morbid obesity, which have also been observed to increase risks of poor outcome from COVID-19 in the general population.

In the MS population within the COViMS Registry and in other registries/surveys, common risk factors for severe COVID-19 outcomes included older age, male sex, Black race, higher disability level, recent corticosteroid use, and use of B cell-depleting therapies. ${ }^{1,2,5}$ No demographic or DMT differences were observed in relation to NMOSD outcomes, although our statistical power to identify differences was limited. Overall, almost $60 \%$ of patients with NMOSD with COVID-19 in COViMS were treated with B cell-depleting therapies (46/77), and the proportion of NMOSD taking rituximab with fatal outcomes was nominally higher compared with those not taking rituximab $(13.0 \%$ vs $6.5 \%)$. In addition, a large proportion of NMOSD were of Black race (29/77), and 4 of the 8 deaths were in NMOSD of Black race. Hence, with a larger sample size, rituximab and Black race could prove to be additional risk factors for severe COVID-19 in NMOSD. In fact, to achieve an $80 \%$ power to detect a difference in more severe outcomes between these groups, it would require over 200 patients per group. Thus, precautions to prevent exposure to SARS-CoV-2 in patients with NMOSD are recommended. ${ }^{6,7}$ Moreover, COVID-19 vaccination has been strongly encouraged by infectious disease experts and the Centers for Disease Control and Prevention, especially for those who are at high risk for more severe COVID-19 disease, and thus COVID-19 vaccination in NMOSD may be particularly important. ${ }^{6,7}$

None of the 20 patients with MOGAD with COVID-19 in the COViMS Registry died, and only 5 required hospitalization. Patients with MOGAD in this cohort had similar comorbidities as those with NMOSD, and almost half were taking rituximab. Not identifying specific risks factors of poorer outcomes with COVID-19 in MOGAD is likely due to the small size of the cohort.

Altering B cell-depleting therapy dosage and/or frequency of administration during the COVID-19 pandemic has been 
Table 4 Differences in COVID-19 Deaths Among Patients With MS and NMO in COViMS

\begin{tabular}{|c|c|c|c|c|}
\hline Factor & Total $(\mathrm{N}=82)$ & MS $(n=74)$ & NMO $(n=8)$ & $p$ Value \\
\hline Sex & & & & $0.21^{\mathrm{b}}$ \\
\hline Male & $35(42.7)$ & $34(45.9)$ & $1(12.5)$ & \\
\hline Female & $46(56.1)$ & $39(52.7)$ & $7(87.5)$ & \\
\hline Nonbinary & $1(1.2)$ & $1(1.4)$ & $0(0.0)$ & \\
\hline Age & $60.9(13.5)$ & $62.1(12.3)$ & $50.1(19.2)$ & $0.016^{e}$ \\
\hline Race & & & & $0.18^{\mathrm{b}}$ \\
\hline Non-Hispanic White & $54(65.9)$ & $51(68.9)$ & $3(37.5)$ & \\
\hline Black or African American & $20(24.4)$ & $16(21.6)$ & $4(50.0)$ & \\
\hline Hispanic or Latino & $2(2.4)$ & $2(2.7)$ & $0(0.0)$ & \\
\hline Other/unknown & $6(7.3)$ & $5(6.8)$ & $1(12.5)$ & \\
\hline Country at the time of COVID-19 onset ${ }^{c}$ & & & & $0.99^{\mathrm{b}}$ \\
\hline United States & 77 (97.5) & $70(97.2)$ & $7(100.0)$ & \\
\hline Canada & $1(1.3)$ & $1(1.4)$ & $0(0.0)$ & \\
\hline Other & $1(1.3)$ & $1(1.4)$ & $0(0.0)$ & \\
\hline Disease duration $^{c}$ & $19.3(12.2)$ & $20.6(12.1)$ & $9.3(7.4)$ & $0.012^{\mathrm{e}}$ \\
\hline Ambulatory status ${ }^{c}$ & & & & $0.075^{b}$ \\
\hline Fully ambulatory & $15(20.5)$ & $11(16.9)$ & $4(50.0)$ & \\
\hline Walk with assistance & $20(27.4)$ & $18(27.7)$ & $2(25.0)$ & \\
\hline Nonambulatory & $38(52.1)$ & $36(55.4)$ & $2(25.0)$ & \\
\hline Glucocorticoid during the last 2 months? & & & & $0.45^{\mathrm{a}}$ \\
\hline No & $54(76.1)$ & $48(75.0)$ & $6(85.7)$ & \\
\hline Yes & $6(8.5)$ & $5(7.8)$ & $1(14.3)$ & \\
\hline Unknown & $11(15.5)$ & $11(17.2)$ & $0(0.0)$ & \\
\hline DMT at the time of COVID-19 ${ }^{c}$ & & & & $0.002^{\mathrm{b}}$ \\
\hline Alemtuzumab & $1(1.3)$ & $1(1.5)$ & $0(0.0)$ & \\
\hline Dimethyl fumarate & $4(5.3)$ & $4(5.9)$ & $0(0.0)$ & \\
\hline Glatiramer acetate & $2(2.6)$ & $2(2.9)$ & $0(0.0)$ & \\
\hline Interferon-beta & $1(1.3)$ & $1(1.5)$ & $0(0.0)$ & \\
\hline Mycophenolate & $1(1.3)$ & $1(1.5)$ & $0(0.0)$ & \\
\hline Natalizumab & $3(3.9)$ & $3(4.4)$ & $0(0.0)$ & \\
\hline Ocrelizumab & $19(25.0)$ & 19 (27.9) & $0(0.0)$ & \\
\hline Rituximab & $10(13.2)$ & $4(5.9)$ & $6(75.0)$ & \\
\hline Siponimod & $2(2.6)$ & $2(2.9)$ & $0(0.0)$ & \\
\hline Teriflunomide & $5(6.6)$ & $5(7.4)$ & $0(0.0)$ & \\
\hline Other & $2(2.6)$ & $1(1.5)$ & $1(12.5)$ & \\
\hline None & $26(34.2)$ & $25(36.8)$ & $1(12.5)$ & \\
\hline Rituximab use & & & & $<0.001^{a}$ \\
\hline No & $72(87.8)$ & $70(94.6)$ & $2(25.0)$ & \\
\hline
\end{tabular}


Table 4 Differences in COVID-19 Deaths Among Patients With MS and NMO in COViMS (continued)

\begin{tabular}{|c|c|c|c|c|}
\hline Factor & Total $(\mathrm{N}=\mathbf{8 2})$ & MS $(n=74)$ & NMO $(n=8)$ & $p$ Value \\
\hline Yes & $10(12.2)$ & $4(5.4)$ & $6(75.0)$ & \\
\hline Length of time on current $\mathrm{DMT}^{\mathrm{c}}$ & & & & $0.99^{\mathrm{b}}$ \\
\hline$<6$ months & $1(2.1)$ & $1(2.4)$ & $0(0.0)$ & \\
\hline 6 or more months & $46(97.9)$ & $40(97.6)$ & $6(100.0)$ & \\
\hline Have comorbidities? & & & & $0.99^{\mathrm{b}}$ \\
\hline No & $6(7.3)$ & $6(8.1)$ & $0(0.0)$ & \\
\hline Yes & $72(87.8)$ & $64(86.5)$ & $8(100.0)$ & \\
\hline Unknown & $4(4.9)$ & $4(5.4)$ & $0(0.0)$ & \\
\hline Cancer & $7(8.5)$ & $6(8.1)$ & $1(12.5)$ & $0.67^{\mathrm{a}}$ \\
\hline Cardiovascular disease & $22(26.8)$ & $21(28.4)$ & $1(12.5)$ & $0.34^{a}$ \\
\hline Cerebrovascular disease & $1(1.2)$ & $1(1.4)$ & $0(0.0)$ & $0.99^{\mathrm{b}}$ \\
\hline Chronic kidney disease & $7(8.5)$ & $6(8.1)$ & $1(12.5)$ & $0.67^{\mathrm{a}}$ \\
\hline Chronic liver disease & $3(3.7)$ & $3(4.1)$ & $0(0.0)$ & $0.99^{\mathrm{b}}$ \\
\hline Chronic lung disease & $7(8.5)$ & $5(6.8)$ & $2(25.0)$ & $0.079^{a}$ \\
\hline Chronic neurologic disease & $9(11.0)$ & $8(10.8)$ & $1(12.5)$ & $0.88^{\mathrm{a}}$ \\
\hline Diabetes & $17(20.7)$ & $14(18.9)$ & $3(37.5)$ & $0.22^{\mathrm{a}}$ \\
\hline Hypertension & $43(52.4)$ & $39(52.7)$ & $4(50.0)$ & $0.99^{b}$ \\
\hline Immunodeficiency disease & $4(4.9)$ & $4(5.4)$ & $0(0.0)$ & $0.99^{\mathrm{b}}$ \\
\hline Morbid obesity & $19(23.2)$ & $15(20.3)$ & $4(50.0)$ & $0.058^{a}$ \\
\hline Other & $26(31.7)$ & $25(33.8)$ & $1(12.5)$ & $0.22^{\mathrm{a}}$ \\
\hline Pneumonia & 27 (32.9) & $22(29.7)$ & $5(62.5)$ & $0.061^{a}$ \\
\hline Emergency department visited? & & & & $0.18^{\mathrm{a}}$ \\
\hline Yes & $59(72.0)$ & $51(68.9)$ & $8(100.0)$ & \\
\hline No & $8(9.8)$ & $8(10.8)$ & $0(0.0)$ & \\
\hline Unknown & $15(18.3)$ & $15(20.3)$ & $0(0.0)$ & \\
\hline Hospitalized? & & & & $0.30^{\mathrm{b}}$ \\
\hline Yes & $70(85.4)$ & $63(85.1)$ & $7(87.5)$ & \\
\hline No & $9(11.0)$ & $9(12.2)$ & $0(0.0)$ & \\
\hline Unknown & $3(3.7)$ & $2(2.7)$ & $1(12.5)$ & \\
\hline Admitted to the ICU? & & & & $0.27^{\mathrm{b}}$ \\
\hline Yes & $51(62.2)$ & $45(60.8)$ & $6(75.0)$ & \\
\hline No & $18(22.0)$ & $18(24.3)$ & $0(0.0)$ & \\
\hline Unknown & $13(15.9)$ & $11(14.9)$ & $2(25.0)$ & \\
\hline Ventilation? & & & & $0.21^{\mathrm{b}}$ \\
\hline Yes & $41(50.0)$ & $38(51.4)$ & $3(37.5)$ & \\
\hline No & $22(26.8)$ & $21(28.4)$ & $1(12.5)$ & \\
\hline Unknown & 19 (23.2) & 15 (20.3) & $4(50.0)$ & \\
\hline
\end{tabular}


Table 4 Differences in COVID-19 Deaths Among Patients With MS and NMO in COViMS (continued)

\begin{tabular}{|c|c|c|c|c|}
\hline Factor & Total $(\mathbf{N}=\mathbf{8 2})$ & MS $(n=74)$ & NMO $(n=8)$ & $p$ Value \\
\hline Type of COVID-19 diagnosis & & & & $0.99^{b}$ \\
\hline Laboratory positive & 77 (93.9) & $69(93.2)$ & $8(100.0)$ & \\
\hline Suspected COVID-19, not confirmed & $5(6.1)$ & $5(6.8)$ & $0(0.0)$ & \\
\hline \multicolumn{5}{|c|}{ 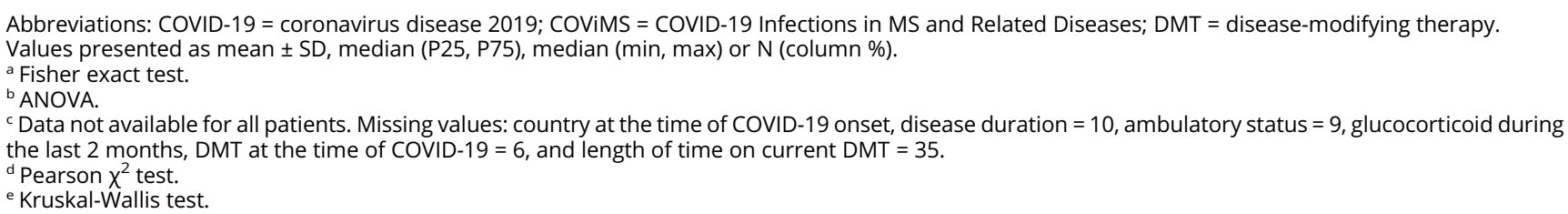 } \\
\hline
\end{tabular}

considered in patients with MS because of the mounting evidence that these agents increase the risk of infections, including COVID-19, in MS. ${ }^{1-3,8}$ Some studies have suggested that patients with MS can achieve similar suppression of new inflammatory disease activity with lower doses and/or extended intervals between doses of rituximab or ocrelizumab. ${ }^{9-12}$ It is unclear whether these treatment approaches would be applicable to NMOSD but may be of interest especially because the main difference in clinical characteristics between patients with NMOSD and MS in COViMS was the use of rituximab. Of interest, 2 small case series in NMOSD have shown that it might be possible to prevent future clinical NMOSD relapses along with reducing the risk of infections by using lower doses of rituximab and/or with a decrease in frequency of rituximab infusions. ${ }^{13,14}$ Moreover, it may be reasonable in some patients (e.g., NMOSD with medical comorbidities and NMOSD with hypogammaglobulinemia) to consider tailoring their treatment regimen in hopes to minimize infectious risks. ${ }^{15}$

Last, in the COViMS Registry, 7 patients with NMOSD who were alive reported having neurologic symptoms in relation to COVID-19 including motor dysfunction (42.9\%) and cognitive and sensory dysfunction (28.6\%). Because of the nature of data collection in the COViMS Registry, it is unclear whether these symptoms were due to a provoked relapse, pseudorelapse, or neurologic complications from COVID-19 as has been seen in other patient populations. ${ }^{16}$ Neurologic symptoms were not reported in the patients with MOGAD in this registry.

Limitations of this study include the small sample size relative to more common diseases (e.g., MS). This study is not population-based, as has been a limitation in many COVID-19 studies. Reporting biases may be present; reporting is voluntary, and more severe outcomes might have been disproportionately captured. Additional risk factors for more severe COVID-19 outcomes might be uncovered with a larger sample size, with studies from areas of the world where NMOSD and MOGAD are particularly prevalent, or when combining data from different registries.

\section{Conclusions}

Among the reported patients with NMOSD, a high mortality rate was observed, and the presence of comorbid conditions was associated with worse COVID-19 outcome. There were no deaths reported in the patients with MOGAD, an observation that was limited due to small sample size.

Overall, these results suggest that it is of utmost importance for people with NMOSD to be especially vigilant and avoid exposure and adopt measures to minimize the risk of infection by SARS-CoV-2. Data will continue to be collected via the COViMS Registry to help enhance our understanding of how infection by SARS-CoV-2 affects both NMOSD and MOGAD.

\section{Acknowledgment}

The COViMS Registry had robust participation from health care professionals across North America (https://www. covims.org/reporter-acknowledgement), and the authors thank all those who contributed information to this effort (see links.lww.com/NXI/A539).

\section{Study Funding}

Support for COViMS is provided by the National MS Society, the Consortium of Multiple Sclerosis Centers, and the MS Society of Canada. REDCap at Washington University in St. Louis is supported by Clinical and Translational Science Award (CTSA) Grant (UL1 TR000448) and Siteman Comprehensive Cancer Center and NCI Cancer Center Support Grant P30 CA091842.

\section{Disclosure}

S.D. Newsome has received consulting fees for scientific advisory boards from Biogen, Genentech, Bristol Myers Squibb, EMD Serono, Novartis, and Greenwich Biosciences; is an 
advisor for Autobahn Therapeutics and BioIncept and a clinical adjudication committee member for a MedDay Pharmaceuticals clinical trial; and has received research funding (paid directly to institution) from Biogen, Novartis, Genentech, National MS Society, Department of Defense, and PatientCentered Outcomes Institute. A.H. Cross received personal compensation from Biogen, Celgene/Bristol Myers Squibb, EMD Serono, Genentech/Roche, Greenwich Biosciences, Janssen Pharmaceuticals, and Novartis and has received research funding (paid directly to institution) from Genentech/ Roche. R.J. Fox has received personal consulting fees from Actelion, Biogen, Celgene, EMD Serono, Genentech, Immunic, Novartis, Sanofi, Teva, and TG Therapeutics. He has served on advisory committees for Actelion, Biogen, Immunic, and Novartis and received clinical trial contract and research grant funding from Biogen and Novartis. J. Halper, P. Kanellis, and B. Bebo report no disclosures. D. Li has received research funding from the Multiple Sclerosis Society of Canada. He is Emeritus Director of the UBC MS/MRI Research Group, which has been contracted to perform central analysis of MRI scans for therapeutic trials with Roche and Sanofi Genzyme. The UBC MS/MRI Research Group has also received grant support for investigator-initiated studies from Sanofi Genzyme, Novartis, and Roche. He has served on the PML-MS Steering Committee for Biogen and given lectures and supported by nonrestricted education grants from Academy of Health Care Learning, Biogen, Consortium of MS Centers, and Sanofi Genzyme. G. Cutter is a member of Data and Safety Monitoring Boards for Astra-Zeneca, Avexis Pharmaceuticals, Biolinerx, Brainstorm Cell Therapeutics, Bristol Meyers Squibb/ Celgene, CSL Behring, Galmed Pharmaceuticals, Horizon Pharmaceuticals, Hisun Pharmaceuticals, Mapi Pharmaceuticals LTD, Merck, Merck/Pfizer, Opko Biologics, OncoImmune, Neurim, Novartis, Ophazyme, Sanofi-Aventis, Reata Pharmaceuticals, Teva pharmaceuticals, VielaBio Inc, Vivus, NHLBI (Protocol Review Committee), and NICHD (OPRU oversight committee). He is on consulting or advisory boards for Biodelivery Sciences International, Biogen, Click Therapeutics, Genzyme, Genentech, GW Pharmaceuticals, KleinBuendel Inc, MedImmune, MedDay, Neurogenesis LTD, Novartis, Osmotica Pharmaceuticals, Perception Neurosciences, Recursion/Cerexis Pharmaceuticals, Roche, and TG Therapeutics. Dr. Cutter is employed by the University of Alabama at Birmingham and President of Pythagoras, Inc., a private consulting company in Birmingham, AL. K. Rammohan receives grant funding from Roche/Genentech, Biogen, EMD Serono, MedDay Pharma, Novartis, TG Therapeutics, and the Department of Defense. He also participates in consulting or advisory groups for Roche/Genentech, Biogen, EMD Serono, and Novartis. A. Salter is a statistical editor for Circulation: Cardiovascular Imaging. Go to Neurology.org/NN for full disclosures.

\section{Publication History}

Received by Neurology: Neuroimmunology \& Neuroinflammation April 1, 2021. Accepted in final form July 6, 2021.

\section{Appendix Authors}

\begin{tabular}{lll}
\hline Name & Location & Contribution \\
\hline $\begin{array}{ll}\text { Scott D. } \\
\text { Newsome, }\end{array}$ & $\begin{array}{l}\text { Johns Hopkins University } \\
\text { So }\end{array}$ & $\begin{array}{l}\text { Drafting/revision of the } \\
\text { manuscript for content, }\end{array}$ \\
& Baltimore, MD & $\begin{array}{l}\text { including medical writing for } \\
\text { content; study concept or } \\
\text { design; and analysis or } \\
\text { interpretation of data }\end{array}$ \\
\end{tabular}

\begin{tabular}{lll}
\hline Anne H. & Washington University in & $\begin{array}{l}\text { Drafting/revision of the } \\
\text { manuscript for content, } \\
\text { Cross, MD }\end{array}$ \\
$\begin{array}{ll}\text { St. Louis School of } \\
\text { Medicine, MO }\end{array}$ & $\begin{array}{l}\text { including medical writing for } \\
\text { content, and analysis or } \\
\text { interpretation of data }\end{array}$ \\
& &
\end{tabular}

\begin{tabular}{lll}
\hline Robert J. & Mellen Center for MS, & $\begin{array}{l}\text { Drafting/revision of the } \\
\text { manuscript for content, } \\
\text { Fox, MD }\end{array}$ \\
& Cleveland Clinic, $\mathrm{OH}$ & $\begin{array}{l}\text { including medical writing for } \\
\text { content, and analysis or } \\
\text { interpretation of data }\end{array}$ \\
\hline
\end{tabular}

June Halper, Consortium of MS Centers, Drafting/revision of the MSN, APN-C Hackensack, NJ manuscript for content, including medical writing for content

\begin{tabular}{lll}
\hline $\begin{array}{l}\text { Pamela } \\
\text { Kanellis, }\end{array}$ & MS Society of Canada, & $\begin{array}{l}\text { Drafting/revision of the } \\
\text { PhD }\end{array}$ \\
& Toronto, Ontario, Canada & $\begin{array}{l}\text { mancript for content, } \\
\text { including medical writing for } \\
\text { content }\end{array}$ \\
\hline
\end{tabular}

\begin{tabular}{lll}
\hline $\begin{array}{l}\text { Bruce Bebo, } \\
\text { PhD }\end{array}$ & $\begin{array}{l}\text { National Multiple Sclerosis } \\
\text { Society (USA), New York, NY }\end{array}$ & $\begin{array}{l}\text { Drafting/revision of the } \\
\text { manuscript for content, } \\
\text { including medical writing for } \\
\text { content }\end{array}$ \\
\hline David Li, MD & $\begin{array}{l}\text { University of British } \\
\text { Columbia, Vancouver, } \\
\text { British Columbia, Canada }\end{array}$ & $\begin{array}{l}\text { Drafting/revision of the } \\
\text { manuscript for content, } \\
\text { including medical writing for } \\
\text { content }\end{array}$ \\
\hline
\end{tabular}

\begin{tabular}{lll}
\hline Gary R. & The University of Alabama \\
Cutter, PhD & at Birmingham & $\begin{array}{l}\text { Drafting/revision of the } \\
\text { manuscript for content, } \\
\text { including medical writing for } \\
\text { content, and analysis or } \\
\text { interpretation of data }\end{array}$ \\
\hline
\end{tabular}

\begin{tabular}{lll}
\hline Kottil W. & University of Miami School & Drafting/revision of the \\
Rammohan, of Medicine, FL & $\begin{array}{l}\text { manuscript for content, } \\
\text { including medical writing for } \\
\text { MD }\end{array}$ & $\begin{array}{l}\text { content } \\
\end{array}$
\end{tabular}

\begin{tabular}{lll}
\hline $\begin{array}{l}\text { Amber } \\
\text { Salter, PhD }\end{array}$ & $\begin{array}{l}\text { Washington University in } \\
\text { St. Louis School of } \\
\text { Medicine, MO }\end{array}$ & $\begin{array}{l}\text { Drafting/revision of the } \\
\text { manuscript for content, } \\
\text { including medical writing for } \\
\text { content; major role in the }\end{array}$ \\
& $\begin{array}{l}\text { acquisition of data; study } \\
\text { concept or design; and } \\
\text { analysis or interpretation of } \\
\text { data }\end{array}$ \\
&
\end{tabular}

\section{References}

1. Salter A, Fox RJ, Newsome SD, et al. SARS-CoV-2 infection in multiple sclerosis patients in North America: relationships of race, ethnicity, comorbidities and disease characteristics to COVID-19 disease course. JAMA Neurol. 2021;78(6):699-708.

2. Sormani MP, De Rossi N, Schiavetti I, et al; Musc-19 Study Group. Disease-modifying therapies and coronavirus disease 2019 severity in multiple sclerosis. Ann Neurol. 2021;89(4):780-789.

3. Louapre C, Maillart E, Papeix C, et al. Outcomes of coronavirus disease 2019 in patients with neuromyelitis optica and associated disorders. Eur J Neurol. 2020. doi: 10.1111/ene.14612. Epub ahead of print. PMID: 33103295.

4. Johns Hopkins University of Medicine Coronavirus Resource Center Mortality Analyses. coronavirus.jhu.edu/data/mortality (accessed 19 March 2021).

5. Safavi F, Nourbakhsh B, Azimi AR. B-cell depleting therapies may affect susceptibility to acute respiratory illness among patients with multiple sclerosis during the early COVID-19 epidemic in Iran. Mult Scler Relat Disord. 2020;43:102195. 
CDC-How to protect yourself and others. cdc.gov/coronavirus/2019-ncov/preventgetting-sick/prevention.html (accessed 19 March 2021).

7. COVID-19 vaccine guidance for people living with MS: nationalmssociety.org/ coronavirus-covid-19-information/multiple-sclerosis-and-coronavirus/covid-19-vaccine-guidance (accessed 19 March 2021).

8. Luna G, Alping P, Burman J, et al. Infection risks among patients with multiple sclerosis treated with fingolimod, natalizumab, rituximab, and injectable therapies. JAMA Neurol. 2020;77(2):184-191.

9. Salzer J, Svenningsson R, Alping P, et al. Rituximab in multiple sclerosis: a retrospective observational study on safety and efficacy. Neurology. 2016;87(20):2074-2081.

10. Nielsen AS, Miravalle A, Langer-Gould A, Cooper J, Edwards KR, Kinkel RP. Maximally tolerated versus minimally effective dose: the case of rituximab in multiple sclerosis. Mult Scler. 2012;18(3):377-378.

11. Maarouf A, Rico A, Boutiere C, et al. Under the aegis of OFSEP. Extending rituximab dosing intervals in patients with MS during the COVID-19 pandemic and beyond? Neurol Neuroimmunol Neuroinflamm. 2020;7(5):e825.
12. Barun B, Gabelić T, Adamec I, et al. Influence of delaying ocrelizumab dosing in multiple sclerosis due to COVID-19 pandemics on clinical and laboratory effectiveness. Mult Scler Relat Disord. 2020;48:102704.

13. Yang CS, Yang L, Li T, et al. Responsiveness to reduced dosage of rituximab in Chinese patients with neuromyelitis optica. Neurology. 2013;81(8): 710-713.

14. Lebrun C, Cohen M, Rosenthal-Allieri MA, et al. Only follow-up of memory B cells helps monitor rituximab administration to patients with neuromyelitis optica spectrum disorders. Neurol Ther. 2018;7(2):373-383.

15. Avouac A, Maarouf A, Stellmann JP, et al. Rituximab-induced hypogammaglobulinemia and infections in AQP4 and MOG antibody-associated, Bertrand Audoin Neurol Neuroimmunol Neuroinflamm. 2021;8(3):e977.

16. Collantes MEV, Espiritu AI, Sy MCC, Anlacan VMM, Jamora RDG. Neurological manifestations in COVID-19 infection: a systematic review and meta-analysis. Can J Neurol Sci. 2021;48(1):66-76. 


\title{
Neurology \\ Neuroimmunology \& Neuroinflammation
}

\author{
COVID-19 in Patients With Neuromyelitis Optica Spectrum Disorders and Myelin \\ Oligodendrocyte Glycoprotein Antibody Disease in North America: From the COViMS \\ Registry \\ Scott D. Newsome, Anne H. Cross, Robert J. Fox, et al. \\ Neurol Neuroimmunol Neuroinflamm 2021;8; \\ DOI 10.1212/NXI.0000000000001057
}

This information is current as of August 24, 2021

\begin{abstract}
Updated Information \&
Services

References

Citations

Subspecialty Collections

Permissions \& Licensing

Reprints

including high resolution figures, can be found at: http://nn.neurology.org/content/8/5/e1057.full.html

This article cites 13 articles, 1 of which you can access for free at: http://nn.neurology.org/content/8/5/e1057.full.html\#\#ref-list-1

This article has been cited by 1 HighWire-hosted articles: http://nn.neurology.org/content/8/5/e1057.full.html\#\#otherarticles

This article, along with others on similar topics, appears in the following collection(s):

All Demyelinating disease (CNS)

http://nn.neurology.org//cgi/collection/all_demyelinating_disease_cns

Information about reproducing this article in parts (figures,tables) or in its entirety can be found online at:

http://nn.neurology.org/misc/about.xhtml\#permissions

Information about ordering reprints can be found online:

http://nn.neurology.org/misc/addir.xhtml\#reprintsus
\end{abstract}

Neurol Neuroimmunol Neuroinflamm is an official journal of the American Academy of Neurology.

Published since April 2014, it is an open-access, online-only, continuous publication journal. Copyright

Copyright $\odot 2021$ The Author(s). Published by Wolters Kluwer Health, Inc. on behalf of the American

Academy of Neurology.. All rights reserved. Online ISSN: 2332-7812.

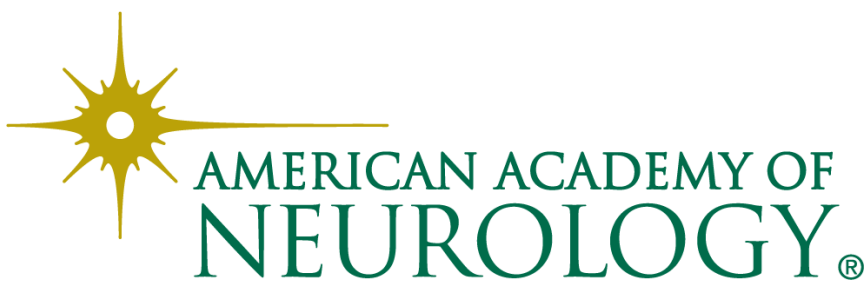

